$\pm N / E$

Global Journals In

(

GLOBAL JOURNAL OF SCIENCE FRONTIER RESEARCH: A

PHYSICS AND SPACE SCIENCE

Volume 20 Issue 10 Version 1.0 Year 2020

Type : Double Blind Peer Reviewed International Research Journal

Publisher: Global Journals

Online ISSN: 2249-4626 \& Print ISSN: 0975-5896

\title{
A Theoretical Proof for the Principle of Equivalence
}

By G. Alagar Ramanujam \& D. Padma Priya

N.G.M College

Abstract- In our recent papers [1][2][3]we proposed two axioms for the primordial Space and using them we derived Newton's law of gravity without using Kepler's laws of planetary motion. Using our axioms, we were able to obtain cosmological equations with dark energy built-in[4]. We show here that the Principle of Equivalence which is the base for Einstein's General theory of relativity follows from our axioms. Based on the day to day common experience Aristotle declared that the time taken by an object dropped at a height to reach the ground will depend upon the heaviness of the object; the more the object is heavy, the lesser the time it takes. After about 2000 years, Galileo took a different view and declared that the time taken by a dropped body to reach the ground is independent of its heaviness. His declaration was against the popular belief and hence he had to establish the truth of his declaration by his famous experiment from the tower of Pisa. After Galileo, several experiments with advanced techniques have confirmed time and again the result that the time taken by a dropped body to reach the ground at a height is independent of its heaviness. We show here that our axioms for Space lead us to a theoretical proof for the above experimental result, which in turn leads to the 'Principle of Equivalence'.

Keywords: principle of equivalence, axioms for space, mass formula, derivation of law of gravity, dark energy.

GJSFR-A Classification: FOR Code: 020399p

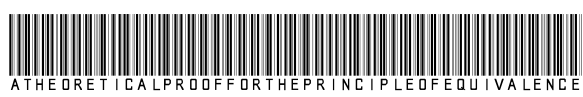

Strictly as per the compliance and regulations of:

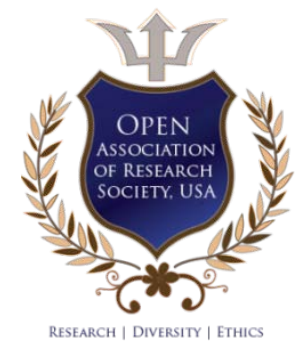

(c) 2020. G. Alagar Ramanujam \& D. Padma Priya. This is a research/review paper, distributed under the terms of the Creative Commons Attribution-Noncommercial 3.0 Unported License http://creativecommons.org/licenses/by-nc/3.0/), permitting all non commercial use, distribution, and reproduction in any medium, provided the original work is properly cited. 


\title{
A Theoretical Proof for the Principle of Equivalence
}

\author{
G. Alagar Ramanujam ${ }^{\alpha} \&$ D. Padma Priya ${ }^{\sigma}$
}

Abstract- In our recent papers [1][2][3]we proposed two axioms for the primordial Space and using them we derived Newton's law of gravity without using Kepler's laws of planetary motion. Using our axioms, we were able to obtain cosmological equations with dark energy built-in[4]. We show here that the Principle of Equivalence which is the base for Einstein's General theory of relativity follows from our axioms. Based on the day to day common experience Aristotle declared that the time taken by an object dropped at a height to reach the ground will depend upon the heaviness of the object; the more the object is heavy, the lesser the time it takes. After about 2000 years, Galileo took a different view and declared that the time taken by a dropped body to reach the ground is independent of its heaviness. His declaration was against the popular belief and hence he had to establish the truth of his declaration by his famous experiment from the tower of Pisa. After Galileo, several experiments with advanced techniques have confirmed time and again the result that the time taken by a dropped body to reach the ground at a height is independent of its heaviness. We show here that our axioms for Space lead us to a theoretical proof for the above experimental result, which in turn leads to the 'Principle of Equivalence'.

Keywords: principle of equivalence, axioms for space, mass formula, derivation of law of gravity, dark energy.

\section{INTRODUCTION}

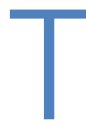
hrough his formula $F=$ ma, Newton brought out the capacity of mass to offer resistance to a force applied on it. The greater the mass is, the lesser is the effect produced on it by an applied force. Einstein through his formula $E=m c^{2}$ brought out the energy equivalent of a given Mass. But in both the cases the question 'What is mass?' remains unanswered. While we know what mass does, we do not know what mass is? From our axioms for the Space, we obtained a formula for mass in terms of the factors that contribute to the mass [1]. By using this mass formula, we obtain here a theoretical proof for the Principle of Equivalence. We prove here that the gravitational acceleration of an object say $B$ towards another object $A$ is completely independent of the massiveness of object $\mathrm{B}$.

From the days of Galileo up to a few years ago, several experiments with most sophisticated techniques have clearly confirmed the Principle of Equivalence. Below given are details of few such experiments. [5]

Author a: Former Principal and Professor of Physics. N.G.M college, Pollachi.India.e-mail: gravity2003@gmail.com

Author o: Founder, Agile Bodhi Institute, Chennai, Tamilnadu, India.
To quote the reference [5]:"Galileo apparently deduced it by timing balls falling along descending slopes...Yet Newton verified it as early as 1687, by observing that pendulums of equal length with bobs made of different materials indeed swung at the same speed, with a precision to three decimal points.

In 1889, using more sophisticated pendulums (torsion pendulums), the Hungarian physicist Loránd Eötvös confirmed it to eight decimal points. In 2008, the US Eöt-Wash group of physicists refined the Principle of the torsion pendulum with beryllium and titanium, and was able to reach a precision to thirteen decimal points, the current record!"

During the Summer of 1971 , on the Moon, the US astronaut David Scott drops a feather and a hammer on the Moon floor, and both reach the ground simultaneously.

The goal of CNES microsatellite, Microscope 2 is to verify the Principle of Equivalence with a precision to fifteen decimal points."

We give below a theoretical proof for the experiment of Galileo.

\section{Proof for the Principle of EQUivalence}

Following the spirit of Le-Sage,[7] we obtain here a proof for Principle of Equivalence. Around the year 1748 Louis Le Sage developed a theory called kinetic theory of gravity wherein the universe was assumed to contain a field of randomly moving tiny particles called ultra-mundane corpuscles with special properties assigned.

An isolated object $A$ is struck by these corpuscles equally from all sides resulting in an inward directed pressure but no net directional pressure. With the second object $B$ present, a fraction of corpuscles that would otherwise have struck $A$ from the direction of B, is intercepted. So, B works a shield. Similarly, the object A works as a shield for the corpuscles that would have normally struck $B$. Because of these shielding effects of different values, the corpuscles drive $A$ and $B$ towards each other. Thus, the apparent attraction between the bodies becomes in this theory, the corpuscles pushing the objects towards each other. The kinetic theory of gravity later on came to be called push gravity or Le sage gravity. In a similar theory presented 
here we have replaced the ultra-mundane corpuscles by the Space.

Our axioms for Space are as follows:

1. Space is all-pervading and is endowed with potential energy. It has the property of constant selfcompression and continually exerting compressive pressure on every system in it.

2. Self-compression results in the formation of infinitesimal spinning quanta of Space, called "formative dust". Due to the surrounding compressive pressure of Space, dust are pressured into formation of discrete groups we know as fundamental particles; every group of dust formed by the surrounding compressive pressure of Space has a spin and hence becomes a source of a radial field with a repulsive pressure at every space-time point.

Explaining our axioms, we state the following: The self-compression of Space results in the infinitesimal spinning quanta of Space called dust. A group of dust formed by the compressive pressure of Space becomes a fundamental particle with spin. From a spinning fundamental particle, energetic constituent dust are thrown out. Each such thrown out dust produces an outgoing wave in Space. These waves produced by every fundamental particle, constitute the Dark energy. The dark energy waves from one particle try to push the other particles away. Like gravity, Dark Energy is present everywhere. The role of Dark Energy is anti-gravity.[1]

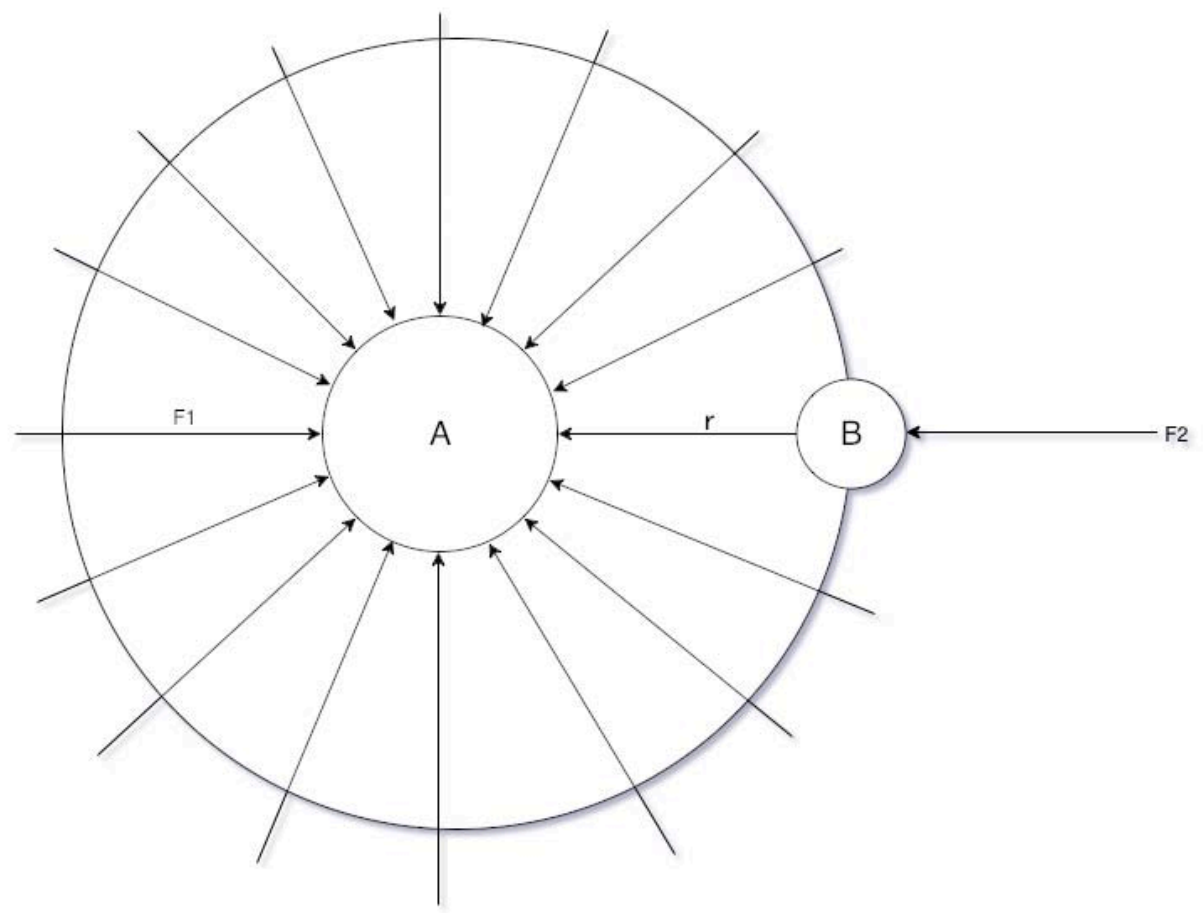

Fig. 1

Let $\mathrm{C}_{1}$ be the compressive pressure on the Unit area of an object (A) due to the Space. Let $R_{1}$ be the repulsive pressure per unit area of the surface of the object $A$ due to the spin of the particles constituting the object $A . C_{1}-R_{1}$ is the net compressive pressure on the object. The total net compressive thrust on the object will be $A 1\left(C_{1}-R_{1}\right)$. Let us denote the net compressive thrust on the first object by the letter $\mathrm{G}_{\mathrm{s}}^{1}$.

$$
\mathrm{G}_{\mathrm{s}}{ }^{1}=\mathrm{A}_{1}\left(\mathrm{C}_{1}-\mathrm{R}_{1}\right) \text { Newton }
$$

The greater the grip is, greater will be the difficulty for a pressure applied on the object to move it. Thus, massiveness of the object or the mass of the object (M) is directly proportional to the grip on the object. Therefore
$M_{1}=\beta G_{s}{ }^{1}$ where $\beta$ is a constant and its unit is $\mathrm{kg} / \mathrm{N}$.

The above formula for mass is called 'The Vethathirian mass formula'.

Here, mass of the object is a consequence of the grip on the object due to the Space. It must be noted here that mass is not an inherent property of the object. But it is a property imposed on the object by the Space.

The total thrust, $A_{1}\left(C_{1}-R_{1}\right)$ converging on the object A,passes through the sphereof radius $r$ (Fig.1). Therefore, the thrust passing through unit area of the sphere will be

$$
A_{1}\left(C_{1}-R_{1}\right) / 4 \pi r^{2}
$$


If we place an object $B$ at a distance $r, B$ will experience a force $F_{2}$ pushing it towards $A$ due to the thrust passing through the sphere.

The pushing force $F_{2}$ due to Space on $B$ is directly proportional to $A_{1}\left(C_{1}-R_{1}\right) / 4 \pi r^{2}$.

Hence $F_{2}=k_{2} A_{1}\left(C_{1}-R_{1}\right) / 4 \pi r^{2}$ where $k_{2}$ is a factor depending upon the nature of the object $\mathrm{B}$.

The factor $k_{2}$ can be considered as the effective area offered by the object $B$, through which the thrust passes before it converges on the object $A$.

$F_{2}$ is the force acting on object $B$ due to the Space. By Newtons third law, as a reaction to $F_{2}$ we have $F_{1}$ due to Space acting on the object $A$. By symmetry, we have

$$
F_{1}=k_{1} A_{2}\left(C_{2}-R_{2}\right) / 4 \pi r^{2}
$$

where $k_{1}$ is the effective area offered by the object $A$.

Because of the forces $F_{1}$ and $F_{2}$ on the objects $A$ and $B$ due to Space, the objects will move towards each other.

Since Space is invisible for us and since Space was considered as vacuum in Newton days the cause for the motion of $B$ towards $A$ was placed on the object $A$ and it was declared that a particle attracts another particle. As explained above it is the Space that gives both the forces $F_{1}$ and $F_{2}$ such that $A$ and $B$ move towards each other. The force with which the object $B$ moves towards $A$ is called gravitational force and as explained above, Space is the cause for the gravitational force.

Since $F_{1}$ and $F_{2}$ are equal according to Newton's third law,

$$
\begin{aligned}
& \mathrm{k}_{2} \mathrm{~A}_{1}\left(\mathrm{C}_{1}-\mathrm{R}_{1}\right) / 4 \pi r^{2}=\mathrm{k}_{1} \mathrm{~A}_{2}\left(\mathrm{C}_{2}-\mathrm{R}_{2}\right) / 4 \pi \mathrm{r}^{2} \\
& \left.\mathrm{k}_{1} \mathrm{~A}_{2}\left(\mathrm{C}_{2}-\mathrm{R}_{2}\right)\right)=\mathrm{k}_{2}\left(\mathrm{~A}_{1}\left(\mathrm{C}_{1}-\mathrm{R}_{1}\right)\right) \\
& \mathrm{k}_{1} / \mathrm{k}_{2}=\mathrm{A}_{1}\left(\mathrm{C}_{1}-\mathrm{R}_{1}\right) / \mathrm{A}_{2}\left(\mathrm{C}_{2}-\mathrm{R}_{2}\right)
\end{aligned}
$$

Solving the above eqn., we get

$$
\begin{aligned}
& \mathrm{k}_{1}=\mu(\mathrm{r}, \mathrm{t})\left(\mathrm{A}_{1}\left(\mathrm{C}_{1}-\mathrm{R}_{1}\right)\right) \\
& \mathrm{k}_{2}=\mu(\mathrm{r}, \mathrm{t})\left(\mathrm{A}_{2}\left(\mathrm{C}_{2}-\mathrm{R}_{2}\right)\right)
\end{aligned}
$$

where $\mu$ is a factor and is a function of $(r, t)$ and its unit is $\mathrm{L}^{2} \mathrm{~N}^{-1}$

$$
\begin{aligned}
& F_{1}=\mu A_{1}\left(C_{1}-R_{1}\right) A_{2}\left(C_{2}-R_{2}\right) / 4 \pi r^{2} \\
& F_{2}=\mu A_{2}\left(C_{2}-R_{2}\right) A_{1}\left(C_{1}-R_{1}\right) / 4 \pi r^{2} \\
& F_{2} / A_{2}\left(C_{2}-R_{2}\right)=\mu A_{1}\left(C_{1}-R_{1}\right) / 4 \pi r^{2}
\end{aligned}
$$

Using eqn.2 we have,

$$
\mathrm{F}_{2} / \mathrm{G}_{\mathrm{s}}^{2}=\mu \mathrm{G}_{\mathrm{s}}^{1} / 4 \pi \mathrm{r}^{2}
$$

The message from eqn.(6) is highly significant. The net grip on object $B$ plays the role of inertia opposing the force $F_{2}$ and the net grip on the object $A$ plays the role of gravity.

Using our mass formula, eqn.(2) we write,

$$
\begin{aligned}
\beta F_{2} / M_{2} & =\mu M_{1} / \beta 4 \pi r^{2} \\
F_{2} / M_{2} & =\mu M_{1} / \beta^{2} 4 \pi r^{2} \\
F_{2} / M_{2} & =G M_{1} / r^{2}=a_{2}
\end{aligned}
$$

where $G(r, t)=\mu / \beta^{2} 4 \pi$ and $a_{2}$ is the acceleration of the object $B$ towards the object $A$.

$G$ is called gravitational constant and its dimension is determined by those of $\mu$ and $\beta$. The dimension of $G$ is $L^{3} \mathrm{~T}^{-2} \mathrm{M}^{-1}$. For Newton, $G$ is a proportionality constant, but for us $G(r, t)$ is a combination of two factors and its dimension emerges from the theory.

The eqn.(7)shows that the acceleration $a_{2}$ of the object $\mathrm{B}$ is completely independent of the nature and the parameters of the object $B$ and thus represents a proof for the Principle of Equivalence.

It is extremely gratifying that our axioms have the potential to lead us to a theoretical proof for the famous experimental results of Galileo. In this connection it is interesting to see the work of Berry [8] and of Paulo Christillin [9] on the concept of the Principle of Equivalence.

\section{ili. Conclusion}

Our axioms for Space, as shown in this paper have the potential to predict the Principle of Equivalence. Einstein used this principle as the base for his general theory of relativity [10]. In the current Physics, since mass is considered as the cause of gravity, there is a necessity to introduce gravitational mass defined as the capacity of an object to attract another object. In the theory presented here, for every object there is only one mass given by our mass formulaeqn. (2). The mass defined by us in eqn.(2) is not only a measure of the inertia of the object but also a measure of the gravitational force between the object $A$ and any other object.

\section{ACKNOWLEDGEMENTS}

We remain very much thankful to Prof. Paolo Christillin of Universita di Pisa, Italy for having drawn our attention to the References (8)and (9) and for his input while preparing the manuscript of this paper. One of us (G.A.R) expresses his deep sense of reverence to Shri. Vethathiri Maharishi for a number of personal discussions on the nature of Space and gravity. We sincerely thank Arulnidhi Uma Vethathiri, Arulnidhi Mr. Vijay Arora (USA) and Arulnidhi Mr. Raj Taneja (USA) for the very useful discussions and encouragement. It is a pleasure to thank Mr. Devarajan, Mr.Gokula Krishnan of Chennai and Mr. Vikram Kannan, Mr. Babu Prasath of Pollachi for their interest in this work. 


\section{References Références Referencias}

1. Alagar Ramanujam, G., Keith Fitzcharles, Muralidharan,S. Physics behind the Dark Matter, Dark Energy and the inflationary expansion of the universe: Indian J. of Phys 93, 959-963, https://doi.org/10.1007/s12648-018-01364-9 (2019).

2. Vijay Arora, Raj Taneja, G. Keith Fitzcharles, Alagar Ramanujam. On The Salient Features of The Vethathirian Cosmology: Int. J. Trend. In. Reasearch and Develop. 6(4), 472(2019)www.ijtrd.com/papers/ IJTRD20708.pdf

3. Alagar Ramanujam, G, Keith Fitzcharles, Muralidharan, S. An Extended Version of Hubble's Law,J.Modern Phys. 8,1067(2017).

4. Alagar Ramanujam, G. Friedmann-Like Cosmological Equations for the Accelerated Expansion of the Universe, J. Modern Phys.11, 996-1004. https://doi.org/10.4236/jmp.2020.117062 (2020).

5. Jean-Philippe Braly. https://news.cnrs.fr/articles/theprinciple-of-Equivalence-put-to-the-test (2016).

6. Nyambuya, Golden. On a Theoretical Proof of the Weak Equivalence Principle from within the Confines of Newtonian Gravitation (2011).

7. Aronson, S. "The gravitational theory of GeorgesLouis Le Sage", The Natural Philosopher, 3: 51-74, (1964).

8. M. Berry, Principles of Cosmology and Gravitation, Cambridge University Press, Cambridge, (1976).

9. P. Christillina, Eur. Phys J. Plus 129, 175(2014), DOI: 10.1140/epjp/i2014-14175-2.

10. Albert Einstein. Relativity: The Special and General theory, Henry Holt and company (1920). 